\title{
Review Paper: Ethical Patient Prioritization in Disaster Triage: A Protocol for a Systematic Review
}

\author{
Vahid Ghanbari ${ }^{1}$, Armin Zareiyan², Amir Nejati ${ }^{1}$, Dan Hanfiling ${ }^{3}$, Ali Ardalan ${ }^{1 *}$ (D \\ 1. Department of Health in Disaster and Emergencies, School of Public Health, Tehran University of Medical Sciences, Tehran, Iran. \\ 2. Department of Health in Emergencies and Disaster, Faculty of Nursing, Islamic Republic of Iran Army University of Medical Sciences, Tehran, Iran. \\ 3. Center for Health Security, University of Pittsburgh Medical Center, Baltimore, The United States of America.
}

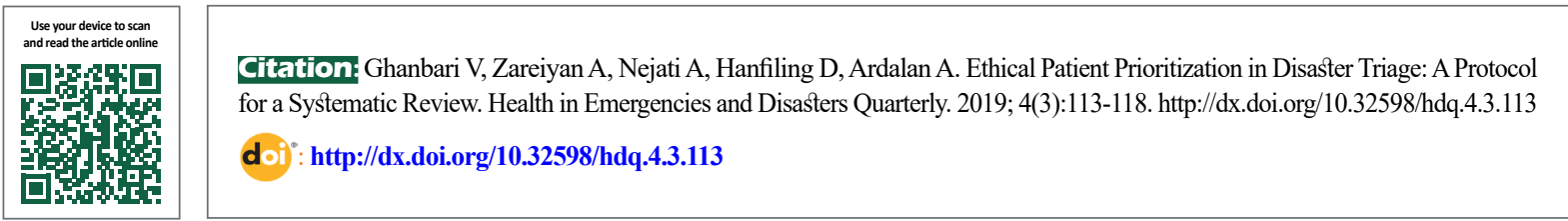

\section{(i) \$)}

Article info:

Received: 10 Oct 2018

Accepted: 23 Feb 2019

Available Online: 01 Apr 2019

\section{Keywords:}

Disaster, Patient prioritization, Medical ethics, Systematic review

\section{ABSTRACT}

Background: Disasters are medically defined as events in which the demands for patients' care far exceed the available resources. In such situations, triage and rationing of limited resources are inevitable. A decision regarding triage needs not only scientific guidelines but also an ethical framework and supporting policies. This study aims to provide a comprehensive review of the criteria for ethical decision-making in disasters triage.

Materials and Methods: Medline (Via pubMed.com), Scopus, Web of Science, and ProQuest databases will be searched from 1990 to July 2017 using a defined search strategy. Other search resources include Google Scholar, World Health Library, Global Ethics Library, Gray Literature Report website, and World Health Organization (WHO), which will be searched using a modified search strategy. The manual search will be conducted in two journals with the highest number of retrieved titles in the Scopus search and the reference list of selected articles. Study selection, quality assessment, and data extraction will be done by the first author, and the second reviewer will check the results, and probable disagreements will be resolved through discussion and review by a third reviewer.

Results: This systematic review will identify all factors a triage officer should be considered when he or she would like to make an ethical decision.

Conclusion: Transparency and consistency are two main procedural ethical values of disaster triage. The result of this review could be used to make a consistent decision in disaster triage.

\section{Introduction}

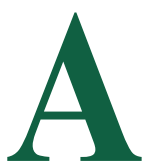

Disaster is a serious event which disturbs the stricken community [1]. In respect of Medical Science, these events cause numerous casualties which their medical needs exceed the capacity of the responders for delivering timely and effective services $[2,3]$. In this situation, the management of available resources has a pivotal role in disaster response outcomes $[4,5]$. Triage can play an important role in addressing the issue of scarce medical resources in these situations $[2,6,7]$.

\footnotetext{
* Corresponding Author:

Ali Ardalan, PhD

Address: Department of Health in Disaster and Emergencies, School of Public Health, Tehran University of Medical Sciences, Tehran, Iran.

E-mail: aardalan@gmail.com
} 
Triage is the process of patient categorization and prioritization based on medical needs and allocation of resources to patients who have a chance to benefit from these resources [4, 7]. Recent evidence suggests that the process of triage is often unofficial or decisions are taken in an ad hoc fashion, and its practical aspects are implemented in different ways [8-10]. Hence, those who perform triage in disasters face a tough decision as to who should receive limited life-saving treatments or who may not benefit from such care $[11,12]$. Nonetheless, life and death are the consequences of decisions in triage [12]. Therefore, decisions made in triage can put the decision-maker under a great deal of pressure [12].

Triage and allocation of limited resources in public health emergencies are still one of the most challenging issues in disaster medicine [13] because health care providers are not expected to make decisions to restrict the care of the patients or their rights [14]. Relying on ethical criteria for decision-making separates triage from arbitrary biased decisions based on personal judgment [12]. There is also an ethical obligation to have plans for decision-making in triage before an event occurs [11] and to guide clinical decisions during public health emergencies via ethical policies and processes $[11,14]$.

Although several studies have evaluated the criteria of ethical decision-making in disasters, there is no consensus on these criteria. Therefore, this study aims to obtain a comprehensive perspective of the proposed criteria for ethical decision-making in disasters triage.

\section{Materials and Methods}

This systematic review protocol has been submitted to the International Prospective Register of Systematic Reviews (http://www.crd.york.ac.uk/PROSPERO) (Registration Number: CRD42016040102). Preferred Reporting Items for Systematic review and Meta-Analysis Protocols (PRISMA-P) will be used to develop this review protocol [15].

\section{Type of studies}

Researchers will include qualitative and review studies about resource allocation in public health emergencies. We also include case studies or editorials that describe ethical aspects of patient prioritization in disasters. We will exclude studies on resource allocation in health care settings in routine situations. We will also exclude articles that resource allocation, triage, or prioritization is not their main subject and explain these topics as part of disaster ethics. This review will not consider any specific participants or populations. In this review, we will investigate health care delivery to the people affected by a disaster. There is no restriction on the disaster types. Those studies will be assessed that investigated the ethical aspects of triage or prioritization of patients and resource allocation of medical resources in disaster situations.

\section{Information sources and search strategy}

We will search the following electronic databases: PubMed, Web of Science, Scopus, and ProQuest, from 1990 until July 2017. Google Scholar, Global Health Library, Global Ethics library, World Health Organization, and Gray Literature Report will be searched using modified search syntax. We will also browse all issues of two journals with the highest records in the Scopus search from 1990 until July 2017 and will check article title to this review. The reference list of the eligible studies will also be assessed for possible relevant titles.

A search strategy will be developed based on a combination of the following factors: 1 . MeSH terms and keywords related to disasters and ethics; 2 . search strategy of a systematic review which assesses resource allocation in disasters and emergencies [9]. There is no language limitation in the search. The search strategy used for searching electronic databases is available in Appendix 1.

\section{Data collection and extraction}

All titles retrieved from electronic databases and other resources will be imported into an Endnote database. Duplicated references will be deleted, and then one of the reviewers (Vahid Ghanbari) will screen the titles. After that, the title and abstract assessment will be done by the first reviewer, and the second author will check the results. Predefined inclusion and exclusion criteria will be used to assess the full texts of the remaining titles. The excluded studies will be retained in a table with the reason for exclusion. Discrepancies will also be discussed to reach a consensus and, if necessary, the third author (Amir Nejati) will assess opinions and take the final decision about the article. The process of selecting the studies will be documented in a PRISMA flow chart (Figure1).

\section{Quality assessment}

Quality assessment will be performed by an appropriate tool from the International Narrative Systematic Assessment tool (INSA) [16]. This step will be done by the first reviewer, and the second author will check the results. The selected articles will not be excluded in the quality assessment phase. A summary of the quality 


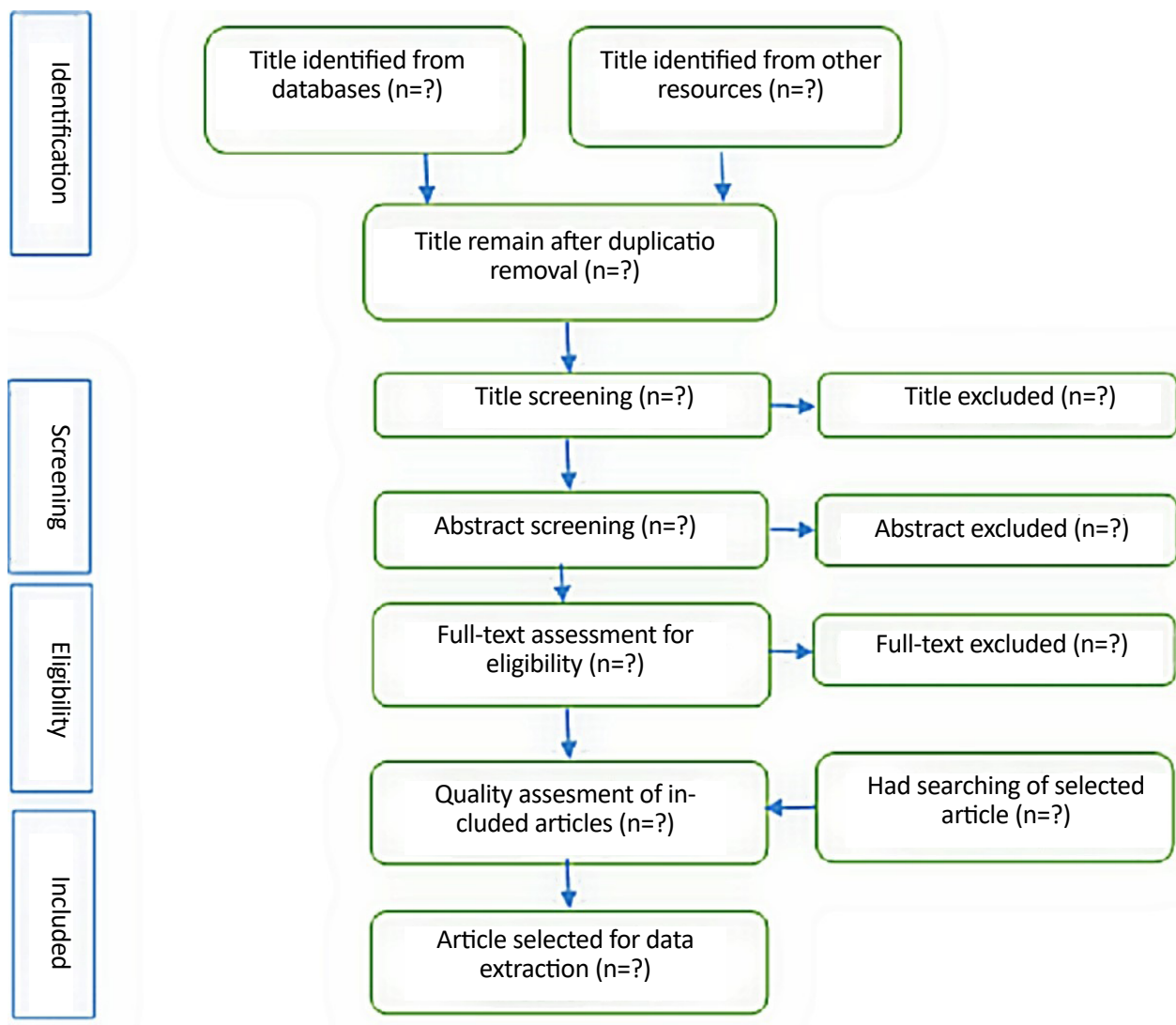

Figure 1. PRISMA diagram for Study selection

assessment scores will be provided. The quality of the evidence will be categorized as high, moderate, and low. Subgroup analysis will not be performed, and publication bias will not be assessed.

\section{Results}

The primary outcome of this review will be factors that physicians or nurses must be considered when they want to decide on patient prioritization. The secondary outcome of this study will be ethical challenges reported in the decision-making process of prioritization of patients in disaster triage. The following data will be extracted from the selected articles: general information (the name of the first author, year, country, type of event), required factors to reach an ethical decision in prioritization of patients in disaster triage and ethical challenges that have been reported at triage, patient prioritization, or resource allocation in disaster. Only qualitative data will be extracted in mixed method studies. Data extraction will be done by Vahid Ghanbari, and the results will be imported into spreadsheets (Microsoft Excel).

\section{Discussion}

Scarcity is the main reason for resource allocation in the health care setting [17]. The shortage of resources worsens in disaster situations [18]. Triage decision-making in disaster events is intended to maximally provide the most benefit for the affected populations [19, 20]. One of the greatest challenges in disaster response is the process of patient prioritization in the context of making triage decisions [21, 22]. Therefore, clinical decisionmaking must be guided by ethical guidelines [21]. This systematic review is expected to provide appropriate evidence for patient prioritization according to ethical principles. Another objective of this study is to address the ethical challenges that care providers face during triage in disasters.

\section{Conclusion}

There are different perspectives regarding how the greatest goods for the greatest number can be achieved in disaster triage. To reach transparency and consistency in a disaster situation, the decision-making criteria should be clear. By identifying and applying these criteria in decision making, not only these ethical values 
will be respected, but also the moral distress which triage officer may experience during or after a decision will be decreased.

\section{Ethical Considerations}

Compliance with ethical guidelines

All ethical principles were considered in this article. The participants were informed about the purpose of the research and its implementation stages; they were also assured about the confidentiality of their information; Moreover, They were allowed to leave the study whenever they wish, and if desired, the results of the research would be available to them.

\section{Funding}

The present paper was extracted from the $\mathrm{PhD}$ thesis of the first author, Vahid Ghanbari, Department of Health in Disaster and Emergencies, School of Public Health, Tehran University of Medical Sciences.

\section{Authors' contributions}

Conceived the study idea, extract the data: Vahid Ghanbari; developing the study protocol: Ali Ardalan, Amir Nejati, Vahid Ghanbari, Dan Hanfiling; Designing the search strategy and search syntax: Vahid Ghanbari; Performing the search and import the results into endnote: Vahid Ghanbari; Title and abstract screening, full-text selection, check the quality of the selected article: Vahid Ghanbari and Armin Zareiyan; Read and approve the article: All authors.

\section{Conflict of interest}

The authors declared no conflict of interest.

\section{Acknowledgments}

The authors would like to acknowledge the Consultation Unit, Office of Publications and Scientometrics, Tehran University of Medical Sciences for editing of the manuscript in English. Also, the authors appreciate Dr. Alireza Bagheri and Dr. Abasali Keshtkar for their valuable comments.

\section{References}

[1] United Nations Office for Disaster Risk Reduction. Terminology on disaster risk reduction. Washington D. C.: United Nations; 2018

[2] Sztajnkrycer MD, Madsen BE, Alejandro Báez A. Unstable ethical plateaus and disaster triage. Emergency Medicine Clinics of North America. 2006; 24(3):749-68. [DOI:10.1016/j emc.2006.05.016] [PMID]

[3] Thompson AK, Faith K, Gibson JL, Upshur RE. Pandemic influenza preparedness: An ethical framework to guide decision-making. BMC Medical Ethics. 2006; 7(12):1-11. [DOI:10.1186/1472-6939-7-12]

[4] Christian MD, Farmer JC, Young BP. Disaster triage and allocation of scarce resources. In: Geiling JA, Burns SM, Rubinson L, Amundson D, Society of Critical Care Medicine, (editors). Fundamental Disaster Management. Illinois: Society of Critical Care Medicine; 2009.

[5] Gostin LO, Hanfling D. National preparedness for a catastrophic emergency: Crisis standards of care. JAMA. 2009; 302(21):2365-6. [DOI:10.1001/jama.2009.1780] [PMID]

[6] Bayer R, Bernheim RG, Crawley LM, Daniels N, Goodman $\mathrm{K}$, Kass N, et al. Ethical considerations for decision making regarding allocation of mechanical ventilators during a severe influenza pandemic or other public health emergency. Atlanta: Centers for Disease Control and Prevention; 2011.

[7] Christian MD, Hawryluck L, Wax RS, Cook T, Lazar NM, Herridge MS, et al. Development of a triage protocol for critical care during an influenza pandemic. Canadian Medical Association Journal. 2006; 175(11):1377-81. [DOI:10.1503/ cmaj.060911] [PMID] [PMCID]

[8] Guidet B, Hejblum G, Joynt G. Triage: What can we do to improve our practice. Intensive Care Medicine. 2013; 39(11):2044-6. [DOI:10.1007/s00134-013-3063-0] [PMID]

[9] Timbie JW, Ringel JS, Fox DS, Pillemer F, Waxman DA Moore $\mathrm{M}$, et al. Systematic review of strategies to manage and allocate scarce resources during mass casualty events. Annals of Emergency Medicine. 2013; 61(6):677-89.e101. [DOI:10.1016/j.annemergmed.2013.02.005] [PMID]

[10] Allen MB, Jesus J. Disaster triage. In: Jesus J, Grossman SA, Derse AR, Adams JG, Wolfe R, Rosen P,(editors) Ethical problems in emergency medicine: A discussionbased review. New Jersey: John Wiley and Sons; 2012 [DOI:10.1002/9781118292150.ch21]

[11] Hick JL, Hanfling D, Cantrill SV. Allocating scarce resources in disasters: Emergency department principles. Annals of Emergency Medicine. 2012; 59(3):177-87. [DOI:10.1016/j. annemergmed.2011.06.012] [PMID]

[12] Moskop JC, Iserson KV. Triage in medicine, part II: Underlying values and principles. Annals of Emergency Medicine. 2007; 49(3):282-7. [DOI:10.1016/j.annemergmed.2006.07.012] [PMID]

[13] Biddison LD, Berkowitz KA, Courtney B, De Jong COLMJ, Devereaux AV, Kissoon N, et al. Ethical considerations: Care of the critically ill and injured during pandemics and disasters: CHEST consensus statement. Chest. 2014; 146(suppl. 4):e145S-55S. [DOI:10.1378/chest.14-0742] [PMID] 
[14] Daniel M. Bedside resource stewardship in disasters: A provider's dilemma practicing in an ethical gap. The Journal of Clinical Ethics. 2012; 23(4):331-5. [PMID]

[15] Moher D, Shamseer L, Clarke M, Ghersi D, Liberati A, Petticrew M, et al. Preferred Reporting Items for Systematic review and Meta-Analysis Protocols (PRISMA-P) 2015 statement. Systematic Reviews. 2015; 4(1):1-9. [DOI:10.1186/20464053-4-1] [PMID] [PMCID]

[16] La Torre G, Backhaus I, Mannocci A. Rating for narrative reviews: Concept and development of the International Narrative Systematic Assessment tool. Senses Sciences. 2015; 2(1):31-5. [DOI: 10.14616/sands-2015-1-3135]

[17] Persad G, Wertheimer A, Emanuel EJ. Principles for allocation of scarce medical interventions. The Lancet. 2009; 373(9661):423-31. [DOI:10.1016/S0140-6736(09)60137-9]

[18] Ytzhak A, Sagi R, Bader T, Assa A, Farfel A, Merin O, et al. Pediatric ventilation in a disaster: Clinical and ethical decision making. Critical Care Medicine. 2012; 40(2):603-7. [DOI:10.1097/CCM.0b013e318232e222] [PMID]

[19] Bottrill MC, Joseph LN, Carwardine J, Bode M, Cook C, Game ET, et al. Is conservation triage just smart decision making? Trends in Ecology \& Evolution. 2008; 23(12):649-54. [DOI:10.1016/j.tree.2008.07.007] [PMID]

[20] Elcioglu O, Unluoglu I. Triage in terms of medicine and ethics. Saudi Medical Journal. 2004; 25(12):1815-9. [PMID]

[21] Kuschner WG, Pollard JB, Ezeji-Okoye SC. Ethical triage and scarce resource allocation during public health emergencies: Tenets and procedures. Hospital Topics. 2007; 85(3):1625. [DOI:10.3200/HTPS.85.3.16-25] [PMID]

[22] Larkin GL. Ethical issues in disaster medicine. In: Koenig KL, Schultz CH, (editors). Koenig and Schultz's Disaster Medicine: Comprehensive Principles and Practices 1. Cambridge: Cambridge University Press; 2009. [DOI:10.1017/ CBO9780511902482.007] 
Appendix 1.

(( Ti=(Ethic*) or Ti=(“Ethical Issues”) or Ti=(“Ethical Issue”) or Ti=(Issue And Ethical) or Ti=(Issues And Ethical) or Ts=("Situational Ethics") or Ts=(Ethics And Situational) or Ts=("Moral Policy") or Ts=("Moral Policies") or Ts=(Policies And Moral) or Ts=(Policy And Moral) or Ts=("Natural Law") or Ts=(Law And Natural) or Ts=(Laws And Natural) or Ts=("Natural Laws") or Ts=(Egoism) or Ts=(Metaethics) or Ts=("Medical Ethic*") or Ts=(Medical And Ethic*) or Ts=("Public Health" And Ethic*) or Ts=("Public Health Ethic*”) or Ts=(Bioethics*)) And (Ti=(Disaster) or Ti=(“Natural Disasters") or Ti=(Disaster And Natural) or Ts=(Disasters And Natural) or Ts=("Natural Disaster") or Ts=("Manmade Disaster*”) or Ts=("Man-Made Disaster*") or Ts=("Man Made" And Disaster) or Ts=("Man-Made" And Disaster*) or Ts=("Casualty Incident*" And Mass) or Ts=(Incident And "Mass Casualty") or Ts=("Mass Casualty Incident") or Ts=("Mass Casualties") or Ts=(Casualty And Mass) or Ts=("Mass Casualty") or Ti=(Emergenc $\left.\left.{ }^{*}\right)\right)$ )

(( $\mathrm{Ti}=($ Ethic*) or Ti=("Ethical Issues") or Ti=(“Ethical Issue”) or Ti=(Issue And Ethical) or Ti=(Issues And Ethical) or Ts=("Situational Ethics") or Ts=(Ethics And Situational) or Ts=("Moral Policy") or Ts=("Moral Policies") or Ts=(Policies And Moral) or Ts=(Policy And Moral) or Ts=("Natural Law") or Ts=(Law And Natural) or Ts=(Laws And Natural) or Ts=("Natural D Laws") or Ts=(Egoism) or Ts=(Metaethics) or Ts=(“Medical Ethic*”) or Ts=(Medical And Ethic*) or Ts=(“Public Health” And $\sum_{0}^{\infty}$ Ethic*) or Ts=(“Public Health Ethic*”) or Ts=(Bioethics*)) And (Ti=(Disaster) or Ti=(“Natural Disasters") or Ti=(Disaster And Natural) or Ts=(Disasters And Natural) or Ts=("Natural Disaster") or Ts=("Manmade Disaster*") or Ts=("Man-Made Disaster*") or Ts=("Man Made" And Disaster) or Ts=("Man-Made" And Disaster*) or Ts=("Casualty Incident" And Mass) or Ts=(Incident And "Mass Casualty") or Ts=("Mass Casualty Incident") or Ts=("Mass Casualties") or Ts=(Casualty And Mass) or Ts=("Mass Casualty") or Ti=(Emergenc*)))

((Title(Ethic*) or Title-Abs(“Ethical Issue*”) or Title-Abs(Issue And Ethical) or Title-Abs(Issues And Ethical) or Title-AbsKey(“Situational Ethics”) or Title-Abs-Key(Ethics And Situational) or Title-Abs-Key(“Moral Policy”) or Title-Abs-Key(“Moral Policies") or Title-Abs-Key(Policies And Moral) or Title-Abs-Key(Policy And Moral) or Title-Abs-Key(“Natural Law”) or TitleAbs-Key(Law And Natural) or Title-Abs-Key(Laws And Natural) or Title-Abs-Key(“Natural Laws”) or Title-Abs-Key(Egoism) or Title-Abs-Key(Metaethics) or Title-Abs("Medical Ethic*”) or Title-Abs(Medical And Ethic*) or Title-Abs("Public Health" And Ethic*) or Title-Abs(“Public Health Ethic*”) or Title-Abs(Bioethics*)) And (Title-Abs(Disaster*) or Title-Abs-Key(“Natural

Disasters") or Title-Abs-Key(Disaster And Natural) or Title-Abs-Key(Disasters And Natural) or Title-Abs-Key(“Natural Disaster") or Title-Abs-Key(“Manmade Disaster"”) or Title-Abs-Key(“Man-Made Disaster*”) or Title-Abs-Key("Man Made” And Disaster) or Title-Abs-Key (“Man-Made” And Disaster*) or Title-Abs-Key(“Casualty Incident*” And Mass) or Title-AbsKey(Incident And "Mass Casualty”) or Title-Abs-Key(“Mass Casualty Incident”) or Title-Abs-Key("Mass Casualties”) or TitleAbs-Key(Casualty And Mass) or Title-Abs-Key (“Mass Casualty") or Title(Emergenc*)) And (Pubyear > 1989))

((Ti(Ethic*) or Ab("Ethical Issue*”) or Ab(Issue And Ethical) or Ab(Issues And Ethical) or Ab("Situational Ethics") or $\mathrm{Ab}$ (Ethics And Situational) or Ab("Moral Policy") or Ab("Moral Policies") or Ab(Policies And Moral) or Ab(Policy And Moral) or Ab("Natural Law") or Ab(Law And Natural) or Ab(Laws And Natural) or Ab("Natural Laws") or Ab(Egoism) or

艹 $A b$ (Metaethics) or Ti(“Medical Ethic*”) or Ti(Medical And Ethic*) or Ab("Public Health" And Ethic*) or Ab("Public Health Ethic*") or Ab(Bioethics*)) And (Ti(Disaster*) or Ab("Natural Disasters") or Ab(Disaster And Natural) or Ab(Disasters And Natural) or Ab("Natural Disaster") or Ab("Manmade Disaster*”) or Ab("Man-Made Disaster*”) or Ab("Man Made" And Disaster) or Ab("Man-Made" And Disaster*) or Ab("Casualty Incident*" And Mass) or Ab(Incident And "Mass Casualty") or Ab("Mass Casualty Incident") or Ab("Mass Casualties") or Ab(Casualty And Mass) or Ab("Mass Casualty”) or $\mathrm{Ti}($ Emergency))) 